\title{
Thiol Suppression of Human Immunodeficiency Virus Type 1 Replication in Primary Cord Blood Monocyte-derived Macrophages In Vitro
}

\author{
Janet Lioy, * Wen-Zhe Ho, ${ }^{\ddagger}$ Joann R. Cutilli, ${ }^{*}$ Richard A. Polin, * and Steven D. Douglas \\ Divisions of *Neonatology and of ${ }^{\ddagger}$ Allergy, Immunology and Infectious Disease, The Children's Hospital of Philadelphia, \\ and Department of Pediatrics, University of Pennsylvania Medical School, Philadelphia, Pennsylvania 19104
}

\begin{abstract}
We investigated the effects of glutathione (GSH), the major naturally occurring thiol, and a pharmacologic thiol precursor of GSH, $N$-acetyl cysteine (NAC), on the expression of human immunodeficiency type 1 (HIV-1) in primary cord blood and adult donor monocyte-derived macrophages (MDM). HIV-1 infection of cord blood and adult MDM was accomplished after incubating 10-15-d-old cultures for $4 \mathrm{~h}$ with a monocyte-tropic strain of HIV-1 (Bal). After 1 wk in culture cell supernatants were tested for reverse transcriptase (RT) activity. MDM were exposed to 5, 10 and $20 \mathrm{mM}$ concentrations of both GSH and NAC before infection, during infection, and after infection was established. GSH and NAC suppressed the replication of HIV1 in both primary cord blood and adult donor MDM in a concentration dependent fashion. These suppressive effects were more pronounced in cord-derived cells than in adult-derived cells. In cells treated with GSH or NAC before infection, there was no significant rise in RT activity as compared with controls. Similarly, when cells were treated with GSH and NAC and simultaneously infected, there was also no significant rise in RT activity after 1 wk in culture. In cells treated after infection was established, RT values were suppressed $80-90 \%$ that of untreated controls. This effect persisted for 1-2 wk after exposure to GSH and NAC. Untreated controls demonstrated syncytium formation and lost characteristics of spreading and elongation 2 wk after HIV-1 infection, whereas most of the treated cells remained free of syncytium and retained cytoplasmic spreading, adherence, and elongation. These data are consistent with other studies of thiol suppression of HIV-1 replication and demonstrate a similar observation for primary cultured cord MDM. These results may offer new approaches toward cellular protection after infection with HIV-1. (J. Clin. Invest. 1993.91:495498.) Key words: $\boldsymbol{\gamma}$-glutamyl amino acids $\bullet \boldsymbol{\gamma}$-glutamylcysteine synthetase - nuclear factor $\mathbf{k B} \cdot$ phorbol myristate acetate • reverse transcriptase
\end{abstract}

\section{Introduction}

Over the last three years there has been an significant increase in the number of pediatric AIDS cases reported by the Centers for Disease Control (1). Although a small number of children have been infected through transfusions of human immunode-

Address reprint requests to Dr. Steven D. Douglas, Section of Immunology, The Children's Hospital of Philadelphia, 34th Street \& Civic Center Blvd., Philadelphia, PA 19104.

Received for publication 21 December 1991 and in revised form 3 September 1992.

J. Clin. Invest.

(C) The American Society for Clinical Investigation, Inc.

0021-9738/93/02/0495/04 \$2.00

Volume 91, February 1993, 495-498 ficiency virus type 1 (HIV-1)-contaminated blood products, $>80 \%$ of infected children have acquired the virus perinatally. Viral transmission can occur prenatally via the placenta, at time of delivery by direct inoculation or postnatally via breast milk. The estimated rates of perinatal transmission is $\sim 30 \%$. Mean survival from the time of diagnosis is estimated at 38 months (1).

The monocyte-derived macrophage $(\mathrm{MDM})^{1}$ may play a central role in the pathogenesis of HIV-1 infection. In vitro the macrophage is believed to contribute to viral latency by acting as a reservoir for replication and dissemination (2-4). We have recently demonstrated that cord MDM are susceptible to HIV1 infection with the monocyte-tropic strains (Bal and Ada-M) and capable of a productive infection (5).

Glutathione (GSH), the most abundant intracellular thiol (0.1-10 mM), plays a key role in intracellular defense against reactive oxygen intermediates and is one of the most active biological compounds available to the cell (6). Roederer et al. (7) have recently demonstrated that $N$-acetyl cysteine (NAC) inhibits tumor necrosis factor- $\alpha$ (TNF- $\alpha$ ) and phorbol myristate acetate (PMA) stimulation of HIV-1 replication in T cells and peripheral blood mononuclear cells. Kalebic et al. (8) have similarly demonstrated dose-dependent suppression of HIV-1 expression in a chronically infected promonocytic U1 cell line with GSH and NAC. These cells were derived from infection of promonocytic U-937 cells carrying two copies of proviral DNA. Recent literature suggests that agents that increase GSH levels inhibit nuclear factor $\mathrm{kB}$ (NF-kB), which is a known enhancer of HIV-1 transcription (9) and is a DNA binding protein responsive to cellular activation. This transcription factor has been shown to enhance HIV-1 replication in T cells after treatment with PMA or TNF- $\alpha$ (10-13). The regulation of this nuclear factor may influence the expression of certain genes responsible for HIV-1 expression. NAC has been shown to block PMA, TNF- $\alpha$, and stimulation of HIV long terminal repeat-directed gene expression (lacZ gene expression) as measured by $\beta$-galactosidase activity ( 7 ). In the asymptomatic adult patient with HIV-1 infection, peripheral blood mononuclear cells, serum, and lung epithelial fluid have reduced levels of GSH and soluble thiols $(14,15)$. This deficiency may be a marker for early transition from latency to the active disease process in the asymptomatic individuals.

This article reports the effect of GSH and NAC on the infectivity of HIV-1 for primary cord blood MDM.

\section{Methods}

Heparinized cord blood (20-50 $\mathrm{U} / \mathrm{ml})$ was obtained from the umbilical vein of healthy term newborn infants after uncomplicated pregnancies and deliveries. All cord blood samples were identified as HIV-1

1. Abbreviations used in this paper: MDM, monocyte-derived macrophage; NAC, $N$-acetyl cysteine; NK-kB, nuclear factor kB. 
antibody negative by anonymous testing with the ELISA method (Abbott Laboratories, North Chicago, IL). Monocytes were purified according to our previously described technique (16). Cord blood was layered on a Ficoll gradient and monocyte fraction separated after adherence to gelatin coated flasks. Cells were plated in $24-w e l l / \mathrm{cm}^{2} \mathrm{cul}-$ ture plates (Corning Glass, Inc., Corning, NY) at a density of $3 \times 10^{5}$ in RPMI containing $30 \%$ fetal calf serum. Monocytes were cultured for 10-15 d when they were considered MDM. After the initial purification, $>97 \%$ of the cells were monocytes as determined by morphology and fluorescence-activated cell sorting (FACS) analysis using monoclonal antibody (mAb) Leu-M3 and low-density lipoprotein specific for MDM (5). In all cases, Limulus amebocyte lysate assay demonstrated that media and reagents were endotoxin-free. HIV-1 strain Bal was isolated from human infant lung tissue and provided by Dr. R. C. Gallo's laboratory through the AIDS Research and Reference Reagent Program, Division of AIDS, National Institute of Allergy and Infectious Diseases, National Institutes of Health, Bethesda, MD. Infection was accomplished by adding $0.2 \mathrm{ml}$ (24-well plate) or $0.05 \mathrm{ml}$ (48-well plate) of HIV-1 (with $5-8 \times 10^{5} \mathrm{cpm} / \mathrm{ml}$ of reverse transcriptase (RT) activity) to each well and incubating the cells for $3 \mathrm{~h}$ at $37^{\circ} \mathrm{C}$. The inoculum was removed and cells were washed four times with RPMI to remove unabsorbed virus. The final wash was tested for RT activity and shown to be free of residual inoculum. Every 5-7 d supernatants were tested for RT activity.

Levels of RT activity present in HIV-1-infected cord MDM were determined by the methods of Willey et al. (17) with modification. In brief, $10 \mu \mathrm{l}$ of culture supernatants was added to $50 \mu \mathrm{l}$ of a cocktail containing poly (A), oligo (dt) (Pharmacia Inc., Piscataway, NJ), $\mathrm{MgCl}_{2}$, Nonidet p-40 and $\left.{ }^{32} \mathrm{P}\right] \mathrm{dTTP}$ (Amersham Corp., Arlington Heights, IL) and incubated for $20 \mathrm{~h}$ at $37^{\circ} \mathrm{C}$. A $30-\mu \mathrm{l}$ portion of the reaction mixture was then spotted on DE 81 paper (Whatman Int., Maidstone, England) and air-dried. The filters were then washed in $2 \times$ standard saline citrate ( $\mathrm{SSC} 0.3 \mathrm{M} \mathrm{NaCl} / 0.03 \mathrm{M}$ sodium citrate, $\mathrm{pH}$ 7.0 ) and $100 \%$ ethanol, dried, cut, and placed in a scintillation counter (LS 7000, Beckman Instruments, Inc., Palo Alto, CA) for measurement of radioactivity.

NAC and GSH were purchased from Sigma Chemical Co. (St. Louis, MO). Cord blood MDM were incubated with NAC and GSH $(5,10$, and $20 \mathrm{mM})$ either $24 \mathrm{~h}$ before adding HIV-1 (Bal), simultaneously with the addition of HIV-1, or within 2 wk of an already established productive infection with HIV-1 (as determined by RT activity). The pretreated cells were exposed to GSH or NAC for a 24-h period before infection with HIV-1. Similarly, cells were simultaneously exposed to GSH or NAC for up to $3 \mathrm{~h}$ during infection with HIV-1 (Bal). In studies of a third group of cells in which infection was already established, fresh GSH or NAC were added at their respective concentrations every $3 \mathrm{~d}$ during the experiment. In the former two groups, GSH and NAC were added only once. RT activity was assessed at 7-10 d postinfection and weekly thereafter for a total of three times. Only those cells which demonstrated cytoplasmic spreading, adherence, and elongation were used for this study. NAC and GSH were dissolved in endotoxin-free media before use.

\section{Results}

In MDM that were infected and then treated, both cord bloodand adult blood-derived cells demonstrated suppression of RT activity after treatment with GSH or NAC in a concentrationdependent fashion (Fig. 1). Although both thiols suppressed viral replication, NAC had a slightly greater effect on $R T$ values than GSH. This suppressive effect was observed 3-5 d after treatment with GSH or NAC, and persisted during $3 \mathrm{wk}$ in culture. After $6 \mathrm{wk}$, both treated and untreated cells demonstrated light microscopic features of cell death (cell detachment loss of spreading and elongation). Uninfected control cells, however, retained characteristics of live macrophage culture,

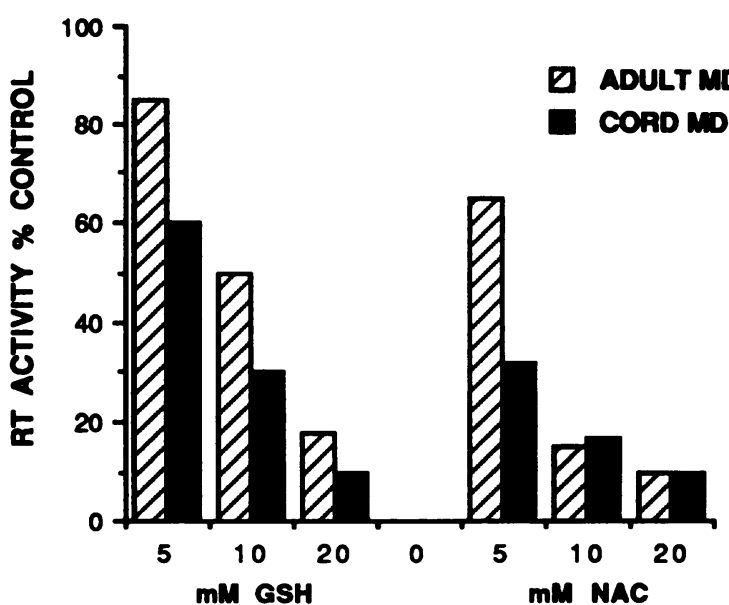

Figure 1. RT suppression of HIV-1 in adult and cord MDM. Concentration-dependent suppression of RT activity with NAC and GSH in cord and adult MDM is shown. RT activity was determined in culture supernatants from the average of two adult and seven cord cell experiments. All results are shown as \% control levels and each experiment was done in triplicate. Variability between triplicate results was $<15 \%$. RT levels for infected control cells averaged 3,000 $\mathrm{cpm} / \mu \mathrm{l}$ corresponding to $100 \%$ activity.

(adherence, spreading, and elongation). Syncytium formation was a constant feature of HIV-1-infected cells and was inhibited by treatment with GSH or NAC. Cord blood-derived MDM also demonstrated higher RT activity compared with adult MDM after both were infected with the same HIV-1 strain (Bal), and greater suppression after thiol treatment (Fig. 2 ). Cells that were pretreated for $24 \mathrm{~h}$ with 10 and $20 \mathrm{mM}$ of GSH or NAC and then infected did not demonstrate significant RT activity after $10 \mathrm{~d}$ in culture. Similarly, cells which were treated and simultaneously infected demonstrated absence of RT activity after $10 \mathrm{~d}$. Treatment with $5 \mathrm{mM} \mathrm{GSH}$ or NAC produced less RT inhibition than with 10 or $20 \mathrm{mM}$ concentrations (Fig. 3, $A$ and $B$ ).

Cell death was seen in all treated and untreated cells $30 \mathrm{~d}$ after infection. The influence of GSH or NAC on cell death was studied by using trypan blue exclusion assay, and there were no differences between the treated and untreated cells during the time course of the experiment.

\section{Discussion}

Glutathione is a ubiquitous molecule present in all mammalian cell systems. It is synthesized and degraded intracellularly, occurring mainly in the reduced form (GSH). The cellular turnover of GSH is associated with its transport in and out of cells via a transpeptidase which breaks down GSH outside the cell membrane, transports its constituent $\gamma$-glutamyl amino acids across into the cell, and resynthesizes GSH inside the cell. The transport of GSH participates in reductive reactions that may occur on the cell membrane as well as the intracellular environment (6). In asymptomatic HIV-1-seropositive individuals, low GSH levels have been found in plasma, peripheral blood mononuclear cells, and lung epithelial lining fluid $(14,15)$.

Cellular protection during oxidant injury from superoxide radical, peroxides and radiation by $\mathrm{GSH}$ is well known $(6,12$, 


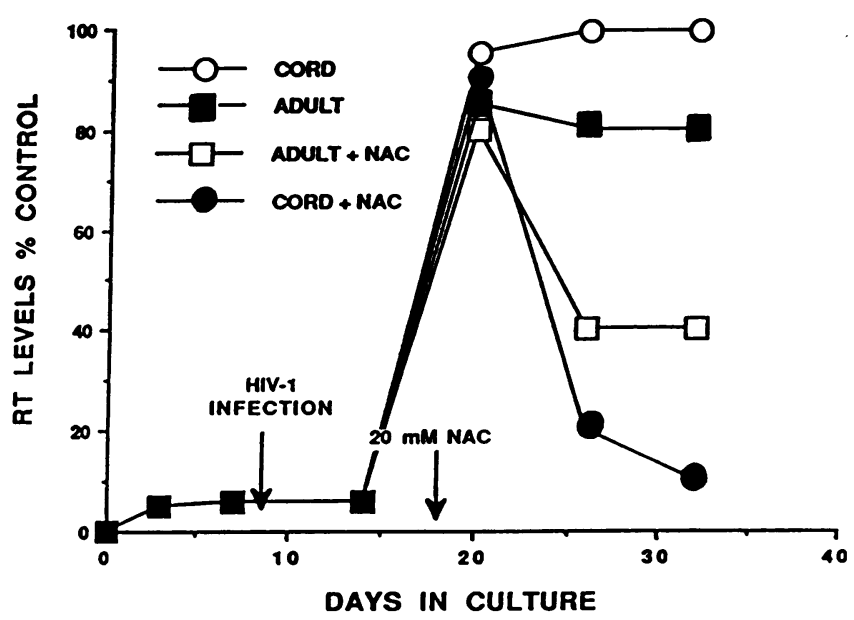

Figure 2. Duration of suppressive effects seen with $20 \mathrm{mM}$ NAC in both cord and adult cells after infection with HIV-1. Results are from the mean of two adult and seven cord experiments done in triplicate. Variability between triplicate results was $<15 \%$. RT levels for infected control cells averaged $3,000 \mathrm{cpm} / \mu \mathrm{l}$ corresponding to $100 \%$ activity. Cell death was seen in both treated and untreated HIV-1-infected cells $30 \mathrm{~d}$ after infection.

$15,18)$. The transport of cysteine sulfur between cells and organs for the reduction of toxic oxygen radical intermediates is a pivotal function of GSH. Depleting GSH in human lymphoid cells markedly increases their sensitivity to radiation $(6,18)$. It has been suggested that this phenomenon is similar to cellular damage due to free radical formation and reactive oxygen intermediate damage. For example, tumor cells also depleted of GSH by buthionine sulfoximine, a $\gamma$-glutamylcysteine synthetase inhibitor, were found to exhibit increased susceptibility to cytolysis after exposure to oxygen radicals (6). Recently, we have demonstrated depletion of GSH levels during superoxide formation after PMA stimulation in cord MDM. Early in the course of PMA stimulation, there is a dramatic decrease in intracellular GSH levels with a slow recovery to baseline levels. (J. Lioy and M. Yudkoff, unpublished data, not shown). GSH is essential not only for its antioxidant function but is also important in the early stages of lymphocyte activation necessary for host defense. For example, the addition of glutathione in vivo enhances activation of cytotoxic T-cells $(19,20)$. Additionally, intracellular glutathione depletion has been found to inhibit mitogen induced lymphocyte activation and natural killer cell function (21).

Repleting intracellular GSH levels by suspending cells in media containing GSH involves the enzymatic degradation, transport across the cell membrane, and resynthesis of GSH inside the cell (6). In cord MDM infected with HIV-1, it is likely that the intracellular GSH pool is increased either by supplying NAC directly to the media or by supplying enough GSH to facilitate breakdown and transport of $\gamma$-glutamyl amino acids into the cell. Increasing the intracellular GSH pool may influence HIV-1 replication in primary cord MDM in a number of ways. Roederer et al. (7) have proposed that oxidative stress caused by PMA or TNF- $\alpha$ stimulation may enhance the binding of the DNA complex protein, NF-kB, thus regulating HIV-1 transcription through gene expression. In the cytoplasm, NF-kB exists in a complex with an inhibitor. After stimulation with PMA, the I-kB component of the cytoplasmic NF-kB complex is phosphorylated allowing its dissociation from the complex, thereby activating HIV-1 transcription within the nucleus. Staal et al. (9) have demonstrated NAC to inhibit the activation of NF-kB, possibly by interfering with phosphorylation or its transport to the nucleus. Similarly, NAC has been shown to suppress transcription of a lacZ gene under control of the HIV promoter and enhancer regulated by NFkB (9).

Our findings observed with primary culture of cord blood MDM may parallel events in vivo. NAC and GSH suppression of HIV-1 in cells which were pretreated suggests that intracellular GSH was increased enough possibly limiting adsorption of the virus through changes on the cell membrane. Similarly, suppression of HIV-1 in cells treated simultaneously while in-
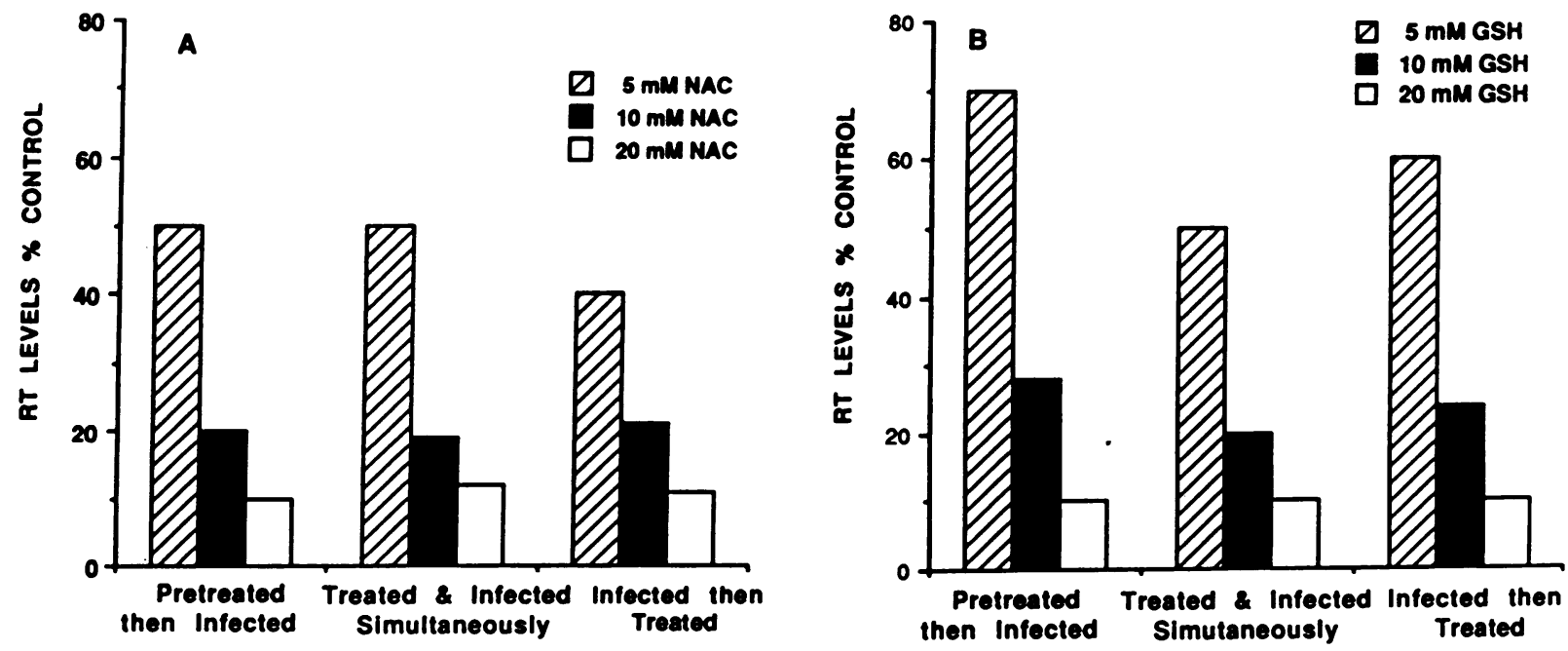

Figure 3. Suppression of RT levels by NAC $(A)$ and GSH $(B)$ during three different conditions in cord cells. More suppression was seen after treatment with higher concentrations $(10$ and $20 \mathrm{mM}$ ) although $5 \mathrm{mM}$ produced some inhibition of RT values. All experiments were done in triplicate. Variability between triplicate results was $<15 \%$. RT levels for infected control cells averaged $3,000 \mathrm{cpm} / \mu \mathrm{l}$ corresponding to $100 \%$ activity. 
process of viral entry into cells. The suppression of cells treated 2 wk after infection was established may imply mechanisms similar to those involving downregulation of transcription activating factor NF-kB or a similar transcription factor responsible for viral replication once the virus has invaded the cell (7-9). In previous work using stimulated and infected PBMC and a $U 1$ cell line, HIV-1 infection was suppressed at concentrations of $5 \mathrm{mM}$ NAC (7). Our study demonstrates a higher concentration dependent response to both NAC and GSH ( 10 and $20 \mathrm{mM}$ ). The use of freshly isolated MDM which are $>97 \%$ purity may account for the decreased sensitivity of these cells to treatment with NAC and GSH.

The mechanisms responsible for differences between cord and adult MDM responses to HIV-1 infection and treatment with thiols remains unknown. We have previously reported that HIV-1 infected cord MDM express higher P24 antigen and RT levels than adult MDM (5). The thiol suppression effect seen in this study is greater for cord than adult MDM (22) infected with HIV-1. Enhanced suppression of viral replication in cord MDM may represent either increased intracellular concentration of NAC and GSH in MDM derived from neonates or greater sensitivity of NF-kB to the suppressive effects of NAC and GSH. Syncytium formation in HIV-1 infected adult and cord MDM has been reported $(5,23)$. Although the mechanism is not known, we observed that NAC and GSH inhibited the formation of such syncytia in cord MDM.

In summary, this study demonstrates the suppressive effects of GSH and NAC on HIV-1 replication in cord MDM. The use of cord blood MDM may provide a close in vitro model in the study of thiol regulation of HIV-1 expression in pediatric AIDS and in the effects of oxidant stress on viral replication and host defense. Finally, the use of thiols in HIV-1 research may provide insight into mechanisms that suppress or prolong latency of the virus.

\section{Acknowledgments}

The authors are grateful to Dr. Marc Yudkoff for providing the authors with expertise in glutathione metabolism. We also thank Ms. Sharon Swink for assistance with monocyte isolation and to Mrs. Peggy McDonald for assistance in preparation of this manuscript.

This investigation was supported by W. W. Smith Charitable Trust grant A4089, NIMH-MH grant 47422, NIH-NS grant 27405, and grants 500-185-11 PG and 500-270-13 PGR, Pediatric AIDS Foundation grants from the American Foundation for AIDS Research.

\section{References}

1. Caldwell, M. B., and M. F. Rogers. 1991. Epidemiology of pediatric HIV infection. Pediatr. Clin. North Am. 38:1-17.

2. Gendelman, H. E., J. M. Orenstein, M. A. Martin, C. Ferrua, R. Mitra, T. Phipps, A. Wahl, C. Lane, S. Fauci, D. S. Burke, et al. 1988. Efficient isolation and propagation of human immunodeficiency virus on recombinant colony-stimulating factor-1 treated monocytes. J. Exp. Med. 167:1428-1441.
3. Gendelman, H. E., J. M. Orenstein, B. Baca, H. Weiser, D. Burger, C. Kalter, and M.S. Melzer. 1989. The macrophage in the persistence and pathogenesis of HIV infection. AIDS (Phila.). 3:475-495. (Editorial Rev.)

4. Pauza, C. D. 1988. HIV persistence in monocytes leads to pathogenesis and AIDS. Cell. Immun. 112:414-424.

5. Ho, Wen-Zhe, J. Lioy, L. Song, J. R. Cutilli, R. Polin, and S. D. Douglas. 1992. Infection of cord blood monocyte-derived macrophages with human immunodeficiency virus type 1 . J. Virol. 66:573-579.

6. Meister, A., and M. E. Anderson. 1983. Glutathione. Annu. Rev. Biochem. 52:711-760.

7. Roederer, M., F. Staal, P. A. Raju, S. W. Ela, A. Herzenberg, and L. Herzenberg. 1990. Cytokine-stimulated human immunodeficiency virus replication is inhibited by $N$-acetyl-L-cysteine. Proc. Natl. Acad. Sci. USA. 87:4884-4888.

8. Kalebic, T., A. Kinter, G. Poli, M. E. Anderson, A. Meister, and A. Fauci. 1990. Suppression of human immunodeficiency virus expression in chronically infected monocytic cells by glutathione, glutathione ester, and $\mathrm{N}$-acetylcysteine. Proc. Natl. Acad. Sci. USA. 88:986-990.

9. Staal, F., M. A. Roederer, A. Herzenberg, and L. Herzenberg. 1990. Intracellular thiols regulate activation of nuclear factor $\mathrm{kB}$ and transcription of human immunodeficiency virus. Proc. Natl. Acad. Sci. USA 87:9943-9947.

10. Nabel, G., and D. Baltimore. 1987. An inducible transcription factor activates expression of human immunodeficiency virus in $\mathrm{T}$ cells. Nature (Lond.). 326:711-713.

11. Griffin, G. E., K. Leung, T. M. Folks, S. Kunkel, and G. Nabel. 1989. Activation of HIV gene expression during monocyte differentiation by induction of NF-kB. Nature (Lond.). 339:70-73.

12. Osborn, L., S. Kunkel, and G. J. Nabel. 1989. Tumor necrosis factor $\alpha$ and interleukin 1 stimulate the human immunodeficiency virus enhancer by activation of the nuclear factor kB. Proc. Natl. Acad. Sci. USA. 86:2336-2340.

13. Duh, E. J., W. J. Maury, T. M. Folks, A. Fauci, and A. B. Rabson. 1989. Tumor necrosis factor $\alpha$ activates human immunodeficiency virus type 1 through induction of nuclear factor binding to the NF-kB sites in the long terminal repeat. Proc. Natl. Acad. Sci. USA 86:5974-5978.

14. Eck, H. P., H. Gmuender, M. Hartmann, D. Petzoldt, V. Daniel, and W. Droege. 1989. Low concentrations of acid-soluble thiol (cysteine) in the blood plasma of HIV-1 infected patients. Biol. Chem. Hoppe Seyler. 370:101-108.

15. Buhl, R., K. J. Holroyd, A. Mastraneli, A. M. Cantin, H. A. Jaffe, F. B. Wells, C. Saltini, and R. G. Crystal. 1989. Systemic glutathione deficiency in symptom-free HIV-seropositive individuals. Lancet. ii:1294-1298.

16. Hassan, N. F., D. Campbell, and S. D. Douglas. 1986. Purification of human monocytes on gelatin-coated surfaces. J. Immunol. Methods. 95:273276.

17. Willey, R. L., D. H. Smith, L. A. Lasky, T. S. Theodore, P. L. Earl, B. Moss, D. Capon, and M. A. Martin. 1988. In vitro mutagenesis identifies a region within the envelope gene of human immunodeficiency virus that is critical for infectivity. J. Virol. 62:139-147.

18. Dethmers, J. K., and A. Meister. 1981. Glutathione export by human lymphoid cells: depletion of glutathione by inhibition of its synthesis decreases export and increases sensitivity to irradiation. Proc. Natl. Acad. Sci. USA 78:7492-7496.

19. Hamilos, D. L., and H. J. Wedner. 1985. The role of glutathione in lymphocyte activation: comparison of inhibitory effects of buthionine sulfoximine and 2-cyclohexene-1-one by nuclear size transformation. J. Immunol. 135:27402747.

20. Droege, W., C. Pottmeyer-Gerber, H. Schmidt, and S. Nick. 1986. Glutathione augments the activation of cytotoxic $\mathrm{T}$ lymphocytes in vivo. Immunohiology. 172:151-156.

21. Fischman, C. M., C. M. Udey, M. Kurtz, and H. J. Wedner. 1981. Inhibition of lectin-induced lymphocyte activation by 2-cyclohexene-1-one:decreased intracellular glutathione inhibits an early event in the activation sequence. $J$. Immunol. 127:2257-2262.

22. Ho, W.-Z., and S. D. Douglas. 1992. Glutathione and $N$-acetylcysteine suppression of human immunodeficiency virus replication in human monocyte/ macrophages in vitro. AIDS Res. Hum. Retroviruses. 8:1249-1253.

23. Collman, R., N. F. Hassan, R. Walker, B. Godfrey, J. R. Cutilli, H. Friedman, S. D. Douglas, and N. Nathanson. 1989. Infection of monocyte derived macrophages with human immunodeficiency virus type 1 (HIV-1). J. Exp. Med. 170:1149-1163. 